\title{
Femtosecond midinfrared study of aggregation behavior in aqueous solutions of amphiphilic molecules
}

\author{
Christian Petersen, ${ }_{1}^{1}$ Artem A. Bakulin, ${ }^{2}$ Vlad G. Pavelyev, ${ }^{2}$ Maxim S. Pshenichnikov, ${ }^{2}$ \\ and Huib J. Bakker ${ }^{1, \text { a) }}$ \\ ${ }^{1}$ FOM Institute for Atomic and Molecular Physics, Science Park 113, Amsterdam, The Netherlands \\ ${ }^{2}$ Zernike Institute for Advanced Materials, University of Groningen, Nijenborgh 4, 9747 AG Groningen, \\ The Netherlands
}

(Received 7 July 2010; accepted 5 September 2010; published online 29 October 2010)

\begin{abstract}
We study the spectral and orientational dynamics of HDO molecules in aqueous solutions of different concentrations of tertiary butyl alcohol (TBA) and trimethylamine- $N$-oxide (TMAO). The spectral dynamics is investigated with femtosecond two-dimensional infrared spectroscopy of the $\mathrm{O}-\mathrm{H}$ stretch vibration of $\mathrm{HDO}: \mathrm{D}_{2} \mathrm{O}$, and the orientational dynamics is studied with femtosecond polarization-resolved pump-probe spectroscopy of the O-D stretch vibration of $\mathrm{HDO}: \mathrm{H}_{2} \mathrm{O}$. Both the spectral and orientational dynamics are observed to show bimodal behavior: part of the water molecules shows spectral and orientational dynamics similar to bulk liquid water and part of the water molecules displays a much slower dynamics. For low solute concentrations, the latter fraction of slow water increases linearly as a function of solute molality, indicating that the slow water is contained in the solvation shells of TBA and TMAO. At higher concentrations, the fraction of slow water saturates. The saturation behavior is much stronger for TBA solutions than for TMAO solutions, indicating the aggregation of the TBA molecules. () 2010 American Institute of Physics. [doi:10.1063/1.3493461]
\end{abstract}

\section{INTRODUCTION}

Most alcohols are well soluble in water, thereby allowing the detailed study of the interactions between water and the hydrophobic groups of alcohols. Frank and Evans ${ }^{1}$ observed that the dissolution of alcohols in water is associated with negative changes in both the enthalpy and the excess entropy $\left(\Delta H<0, \Delta S_{\mathrm{exc}}<0\right)$. Both effects suggest that the introduction of hydrophobic groups leads to an increased structuring of the surrounding water molecules. This structuring was denoted as the formation of hydrophobic icebergs. ${ }^{1}$ This idea was developed in 1945 and is still a topic of intense debate.

A significant contribution to the understanding of the structure of mixtures of alcohols and water comes from neutron scattering experiments ${ }^{2-5}$ that provide time-averaged information on the radial distribution functions of the constituent atoms. Neutron scattering studies on both dilute and concentrated alcohol solutions found the structure of the solvating water to be similar to that of bulk liquid water. ${ }^{2,5}$ Hence, the hydration of alcohols appears not to be accompanied by the formation of an icelike structure of the surrounding water molecules.

Interestingly, the dynamics of water molecules surrounding the hydrophobic groups was observed to be quite different from bulk liquid water. NMR studies, femtosecond midinfrared spectroscopy measurements, and molecular dynamics simulations, all showed that the solvating water

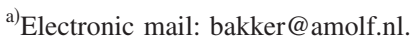

molecules possess significantly slower orientational dynamics than the molecules in bulk liquid water. ${ }^{6-15}$

An interesting property of alcohols and other amphiphilic molecules is that these molecules can form aggregates in aqueous solution as a result of the hydrophobic interaction. This interaction plays an important role in processes such as protein folding and the formation of bilipid membranes. Neutron scattering studies showed that alcohol molecules can form single component aggregates in aqueous solutions. ${ }^{16-20}$ For instance, in a 5\% solution of methanol, the alcohol forms clusters containing 3-8 molecules. ${ }^{17}$ At intermediate alcohol concentrations, evidence was found that methanol and water both form separate percolating molecular networks, ${ }^{19}$ and at high alcohol concentrations, the water molecules are observed to form clusters in the alcohol network. ${ }^{5}$ For a highly concentrated solution of methanol in water (7:3 molar ratio), most of the water is contained in clusters or strings containing 2-20 molecules. ${ }^{5}$

Here, we report on a study of the clustering of tertiary butyl alcohol (TBA) and trimethylamine- $\mathrm{N}$-oxide (TMAO) molecules in aqueous solutions using femtosecond twodimensional midinfrared spectroscopy $(2 \mathrm{DIR})^{21-24}$ and polarization-resolved femtosecond midinfrared spectroscopy. ${ }^{25-29}$ These techniques allow the measurement of the full vibrational frequency-frequency and orientational correlation functions of the water molecules in a solution. ${ }^{12,14}$ Thereby these techniques enable the determination of the fraction of water in solution that solvates the solute molecules. We use these techniques to determine the aggregation behavior of the TBA and TMAO molecules. 


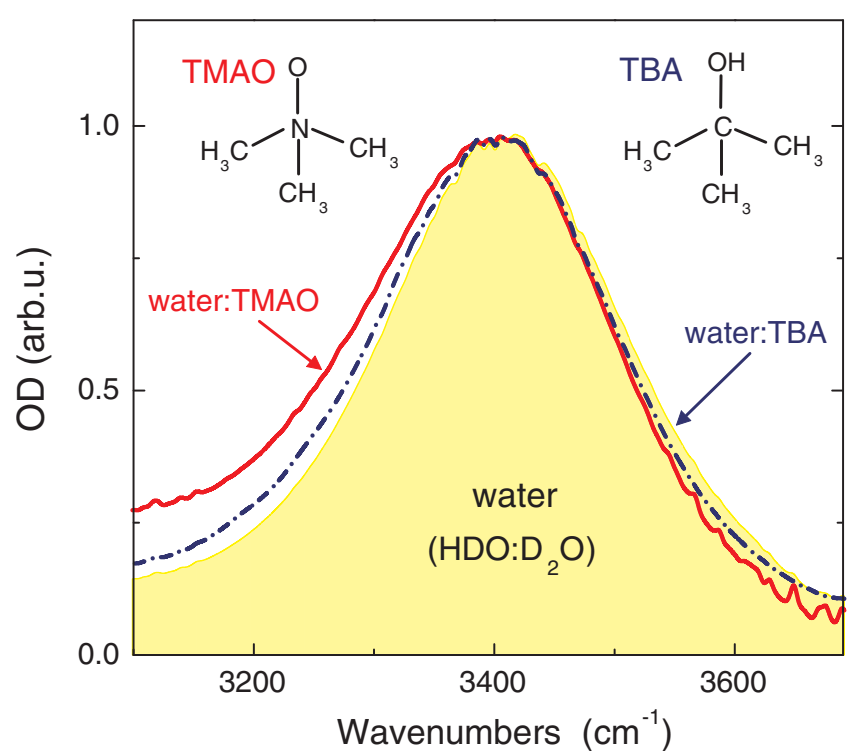

FIG. 1. Structural formulas of TBA and TMAO and the linear absorption spectra of the $\mathrm{O}-\mathrm{H}$ stretch vibration of $\mathrm{HDO}$ for solutions of TBA and TMAO in $4 \%$ HDO: $\mathrm{D}_{2} \mathrm{O}$ and for pure $4 \% \mathrm{HDO}: \mathrm{D}_{2} \mathrm{O}$. The concentration of the solutions is 1 solute molecule per 10 water molecules $(w=0.1)$.

\section{EXPERIMENTAL}

TBA and TMAO are similar in their hydrophobic parts, as both molecules possess three methyl groups connected to a central atom. The hydrophilic group of these solutes is quite different: TMAO possesses a very polar $\mathrm{N}^{+} \mathrm{O}^{-}$group, while TBA possesses a more weakly polar $\mathrm{O}-\mathrm{H}$ group that has a similar polarity as the $\mathrm{O}-\mathrm{H}$ groups of water. The structural formulas of TBA and TMAO are shown in Fig. 1.

We measure the frequency-frequency correlation function of the $\mathrm{O}-\mathrm{H}$ stretch vibration of HDO in solutions of TBA and TMAO in a dilute solution of HDO in $\mathrm{D}_{2} \mathrm{O}$ using two-dimensional infrared (2D-IR) spectroscopy. In all experiments, a low concentration of HDO is used to avoid the measurements being affected by resonant Förster energy transfer between the $\mathrm{O}-\mathrm{H}$ stretch vibrations located on different HDO molecules. ${ }^{30}$ Probing the $\mathrm{O}-\mathrm{H}$ vibration of HDO instead of the O-D vibration has as an advantage that the absorption band of the $\mathrm{O}-\mathrm{H}$ vibration shows less motional narrowing than the $\mathrm{O}-\mathrm{D}$ vibration, thus allowing for a clearer determination of the spectral diffusion dynamics. The $\mathrm{O}-\mathrm{H}$ vibration of $\mathrm{HDO}: \mathrm{D}_{2} \mathrm{O}$ has a strong absorption at $2.9 \mu \mathrm{m}\left(\sim 3400 \mathrm{~cm}^{-1}\right)$. Femtosecond pulses at this wavelength are generated via parametric amplification in $\beta$-barium borate (BBO) and potassium-titanyl phosphate (KTP) crystals $^{31}$ that are pumped with the output of a homebuilt Ti:sapphire laser system. The generated pulses at $3 \mu \mathrm{m}$ have a duration of $\sim 70$ fs and an energy of $\sim 5 \mu \mathrm{J}$.

The 2D-IR experiment is a four pulse experiment in which two pulses are used to generate a frequencymodulated population grating of the excited $\mathrm{O}-\mathrm{H}$ stretch vibration. The third pulse enters after a waiting time $T$ to generate a photon-echo signal. The diffracted echo signal is heterodyne detected by interfering the photon-echo signal with the fourth subpulse and sending the total signal onto an infrared detector array to determine the probe frequency $\left(\omega_{3}\right)$ dependence. The excitation frequency $\omega_{1}$ is obtained by repeating the experiment for many delays $\tau_{12}$ and by performing a Fourier transform of the total signal with respect to this variable. The result is a two-dimensional spectrum that can be presented in a contour plot with the horizontal axis formed by the excitation frequency $\omega_{1}$ and the vertical axis by the probe frequency $\omega_{3}$. A detailed description of this experiment can be found in Ref. 22.

In the polarization-resolved pump-probe experiments, we study solutions of TBA/TMAO and 4\% HDO dissolved in $\mathrm{H}_{2} \mathrm{O}$. In these experiments, we probe the $\mathrm{O}-\mathrm{D}$ vibration of HDO that has a strong absorption around $4 \mu \mathrm{m}$ $\left(2500 \mathrm{~cm}^{-1}\right)$. The anisotropy of the excitation is probed with probing pulses that are polarized parallel and perpendicular to the pump polarization, thus yielding transient absorption changes $\Delta \alpha_{\|}(T)$ and $\Delta \alpha_{\perp}(T)$. These absorption changes are used to construct the so-called anisotropy parameter,

$$
R(T)=\frac{\Delta \alpha_{\|}(T)-\Delta \alpha_{\perp}(T)}{\Delta \alpha_{\|}(T)+2 \Delta \alpha_{\perp}(T)} .
$$

The dynamics of the anisotropy parameter directly reflects the reorientation of the O-D groups of the HDO molecules. The advantage of probing the O-D vibration over the $\mathrm{O}-\mathrm{H}$ vibration in this experiment is that the vibrational lifetime of the O-D vibration $\left(T_{1, \mathrm{O}-\mathrm{D}}=1.8 \mathrm{ps}\right)$ is longer than the vibrational lifetime of the $\mathrm{O}-\mathrm{H}$ vibration $\left(T_{1, \mathrm{O}-\mathrm{H}}=0.7 \mathrm{ps}\right)$. As a result, the dynamics of the anisotropy of the excitation can be probed over a much longer delay time range. The details of this experiment are described in Ref. 13.

The 2D-IR experiments are performed on a thin $\sim 40-60 \mu \mathrm{m}$ film in a wire-guided jet. ${ }^{32}$ The polarizationresolved pump-probe experiments are performed on solutions contained in a cell with a sample thickness of $25 \mu \mathrm{m}$. The concentration of the solute is indicated with the ratio $w$ of the number densities of TBA/TMAO and (HDO $\left.+\mathrm{D}_{2} \mathrm{O} / \mathrm{HDO}+\mathrm{H}_{2} \mathrm{O}\right)$ molecules; $w$ ranges from 0 to 0.2 . The experiments are performed at a temperature of $295 \mathrm{~K}$.

\section{RESULTS}

\section{A. Linear infrared spectra}

Figure 1 shows the linear absorption spectra of the $\mathrm{O}-\mathrm{H}$ band of HDO for pure HDO: $\mathrm{D}_{2} \mathrm{O}$ and for solutions of TBA and TMAO in HDO: $\mathrm{D}_{2} \mathrm{O}$ with $w=0.1$. The frequency of the $\mathrm{O}-\mathrm{H}$ stretch vibration decreases with increasing strength of the local $\mathrm{O}-\mathrm{H} \cdots \mathrm{O}$ hydrogen-bond interaction. The absorption spectrum of the TBA solution is observed to be very similar to that of pure HDO: $\mathrm{D}_{2} \mathrm{O}$, thus showing that neither the hydrophobic part nor the hydrophilic hydroxyl group of TBA has a significant effect on the strength of the waterwater and water-alcohol hydrogen-bond interactions. The $\mathrm{O}-\mathrm{H}$ absorption spectrum of the TMAO solution shows a small redshift, indicating that the average hydrogen bond is somewhat stronger in this solution than in pure HDO: $\mathrm{D}_{2} \mathrm{O}$. This redshift is likely due to the interactions between HDO and the strongly polar $\mathrm{N}^{+} \mathrm{O}^{-}$group of the TMAO molecules. 


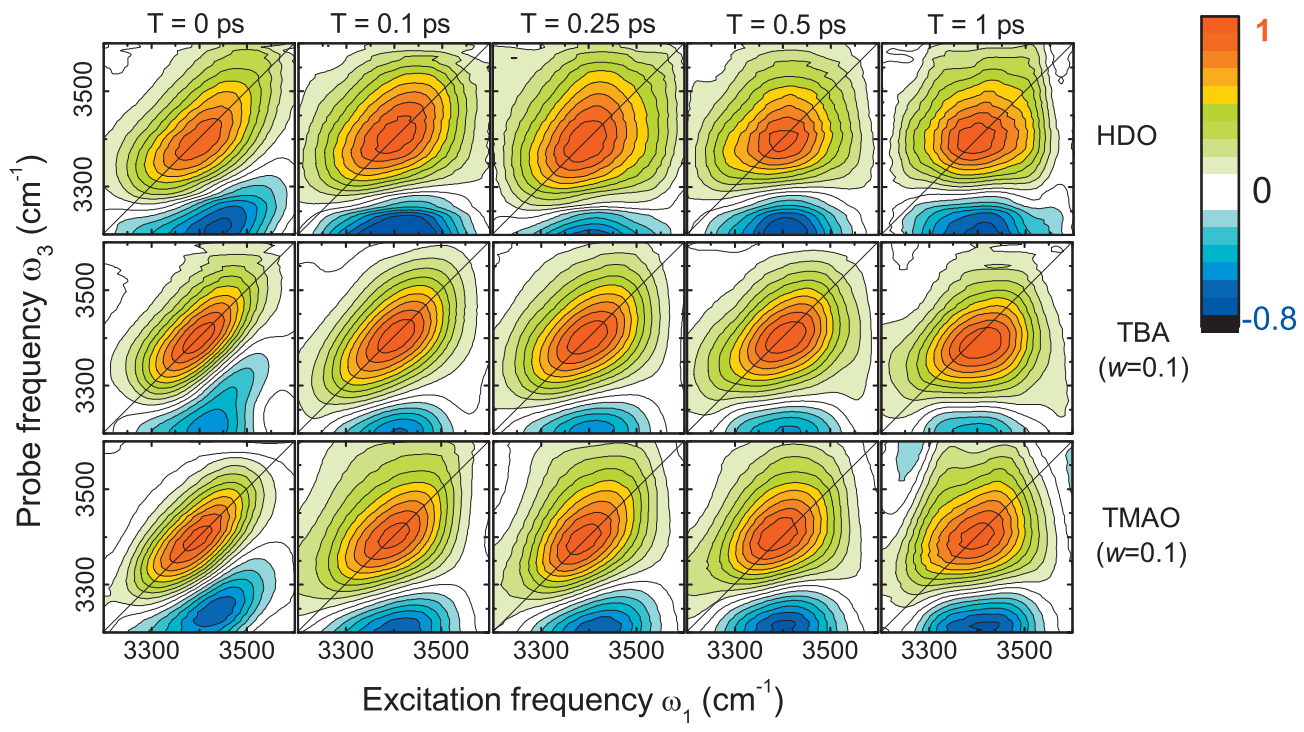

FIG. 2. Two-dimensional vibrational spectra of pure HDO: $\mathrm{D}_{2} \mathrm{O}$ and of solutions of TBA and TMAO in $\mathrm{HDO}: \mathrm{D}_{2} \mathrm{O}$ with $w=0.1$ measured at different waiting times. Positive signals (red color) correspond to the bleaching of the fundamental $v=0 \rightarrow 1$ transition and negative signals (blue color) represent the $v=1$ $\rightarrow 2$ excited state absorption. Each $2 \mathrm{D}$ spectrum is normalized with respect to its maximum. The equilateral contours are drawn with $10 \%$ steps of the maximum amplitude.

\section{B. 2D-IR experiments}

Figure 2 shows the 2D-IR spectra of the $\mathrm{O}-\mathrm{H}$ stretch vibration of $\mathrm{HDO}$ for pure HDO: $\mathrm{D}_{2} \mathrm{O}$ and for solutions of TBA and TMAO with a concentration $w=0.1$. The positive signal at the diagonal of the 2D-IR spectra represents the bleaching of the $v=0 \rightarrow 1$ absorption and the $v=1 \rightarrow 0$ stimulated emission. The negative signal that is shifted with respect to the diagonal along the vertical (probe) axis represents the $v=1 \rightarrow 2$ excited state absorption. This latter signal is located at a lower frequency due to the vibrational anharmonicity.

For all solutions, the 2D-IR spectra display a substantial elongation for waiting times $T$ of 0 and $0.1 \mathrm{ps}$. This indicates that the strength of the hydrogen bonds and the overall structure of the hydrogen-bond network do not experience significant changes at the $\sim 0.1 \mathrm{ps}$ timescale. For pure HDO: $\mathrm{D}_{2} \mathrm{O}$, the spectra start acquiring a more circular shape at a waiting time $T$ of $250 \mathrm{fs}$, and the shape is completely circular at $T$ $=1 \mathrm{ps}$. At this waiting time, the 2D-IR spectra are still elongated along the diagonal for the $w=0.1$ solutions of TBA and TMAO, showing that in these solutions the spectral diffusion of the $\mathrm{O}-\mathrm{H}$ stretch vibrations is slowed down significantly in comparison to pure HDO: $\mathrm{D}_{2} \mathrm{O}$. A similar slowing down of the spectral diffusion has been observed before for aqueous solutions of tetramethylurea. ${ }^{33}$

Figure 3 presents the 2D spectra for highly concentrated solutions of TBA and TMAO with $w=0.2$. In comparison to the solutions with $w=0.1$ (Fig. 2), the dynamics of the TBA solution has not changed much. In contrast, for the TMAO solution, the 2D spectra show a more elongated shape at long $(>0.5 \mathrm{ps})$ waiting times, which reveals a significant further slowing down of the spectral diffusion in this solution.

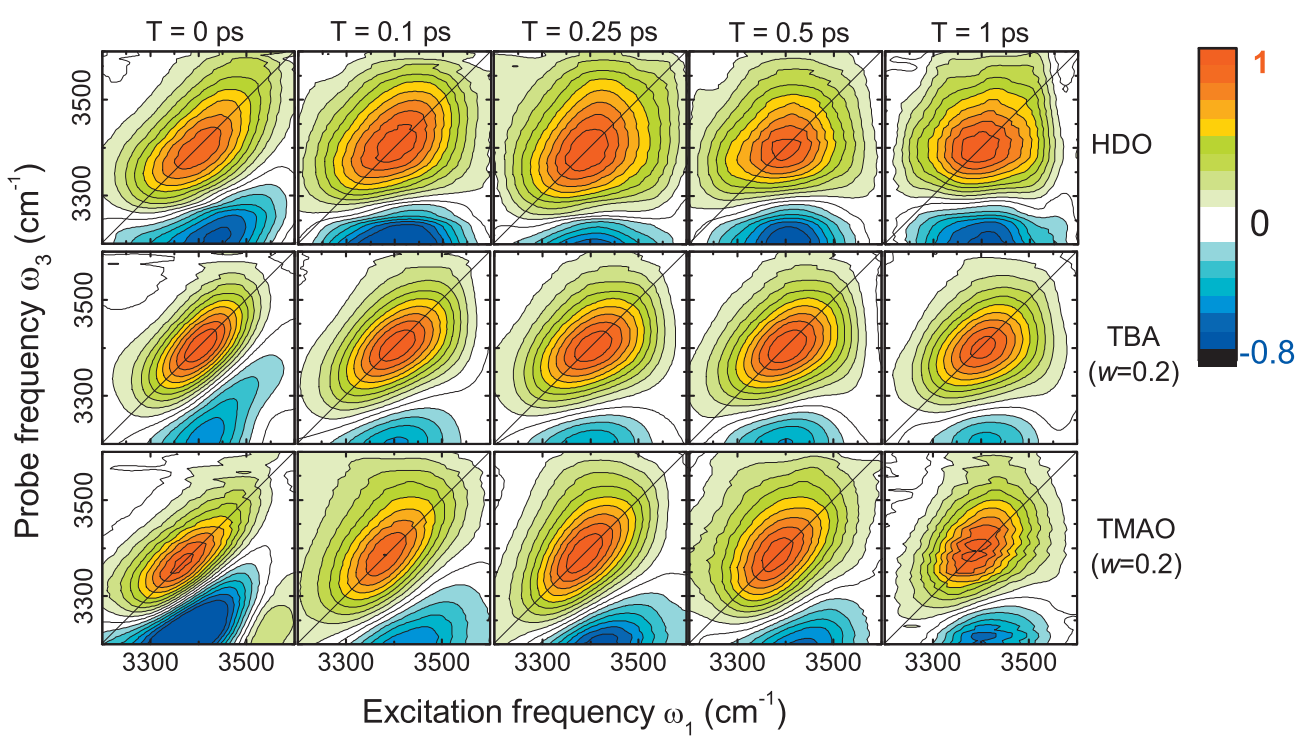

FIG. 3. The same as in Fig. 2 for pure HDO: $\mathrm{D}_{2} \mathrm{O}$ and solutions of TBA and TMAO in HDO: $\mathrm{D}_{2} \mathrm{O}$ with $w=0.2$. 


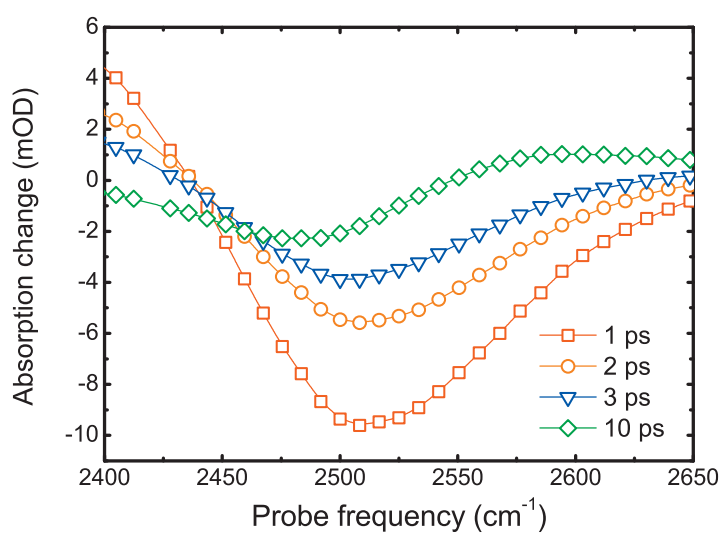

FIG. 4. Transient isotropic spectra at different pump-probe delays of a solution of TBA in HDO: $\mathrm{H}_{2} \mathrm{O}$ with $w=0.1$. The spectra are obtained after exciting the sample with a pump pulse centered at $2500 \mathrm{~cm}^{-1}$. The lines are obtained from a fit to a relaxation model, as described in Ref. 35 .

\section{Polarization-resolved pump-probe experiments}

Figure 4 shows the isotropic transient spectra of the O-D stretch vibration at different pump-probe delays for a TBA solution with $w=0.1$. These transient spectra correspond to an integration over the horizontal (excitation frequency) axis of the 2D spectra that is weighted with the amplitudes given by the spectral shape of the excitation pulse. At early delays, the transient spectra are dominated by the bleaching of the $v=0 \rightarrow 1$ fundamental absorption and $v=1 \rightarrow 0$ stimulated emission. At later delays, the signal is dominated by the change in transmission caused by the redistribution of the excitation energy over the focus. This latter redistribution leads to heating of the sample by a few kelvins and induces a blueshift of the O-D absorption band and a decrease in the absorption cross section. These effects together result in a thermal difference spectrum that shows a bleaching signal at frequencies below $2550 \mathrm{~cm}^{-1}$ and a small induced absorption above this frequency.

The isotropic data [given by the denominator of Eq. (1)] are fitted with a relaxation model in which the O-D stretch vibration relaxes via an intermediate state ${ }^{29}$ to a final thermalized state. The fit to the model is shown with full lines in Fig. 4. From the model, we extract the vibrational relaxation time of the $\mathrm{O}-\mathrm{D}$ vibration $T_{1}$ and the relaxation (thermalization) time of the intermediate state $\tau^{*}$. We find a small increase in $T_{1}$ from $1.8 \pm 0.1$ to $2.0 \pm 0.1 \mathrm{ps}$ and an increase in $\tau^{*}$ from 0.5 to 1 ps going from pure $\mathrm{HDO}: \mathrm{H}_{2} \mathrm{O}$ to a solution of TBA in HDO: $\mathrm{H}_{2} \mathrm{O}$ with $w=0.2$.

In Fig. 5, the parallel and perpendicular transient absorption signals, $\Delta \alpha_{\|}(t)$ and $\Delta \alpha_{\perp}(t)$, are shown as a function of delay for a solution of TMAO with $w=0.2$. These curves represent data taken at a frequency of $2550 \mathrm{~cm}^{-1}$ at which the isotropic thermal signal is equal to zero at all delay times. The figure shows that there exists a long-living difference between the two measured polarizations. The enlargement of the signal at later delays in the right hand panel of the figure reveals that for long delays $\Delta \alpha_{\perp}(t)$ changes from a bleaching signal into an induced absorption. The close-up also shows that the difference between $\Delta \alpha_{\|}(t)$ and $\Delta \alpha_{\perp}(t)$ persists up to delays $>15$ ps. The same effects are observed for TBA solutions.

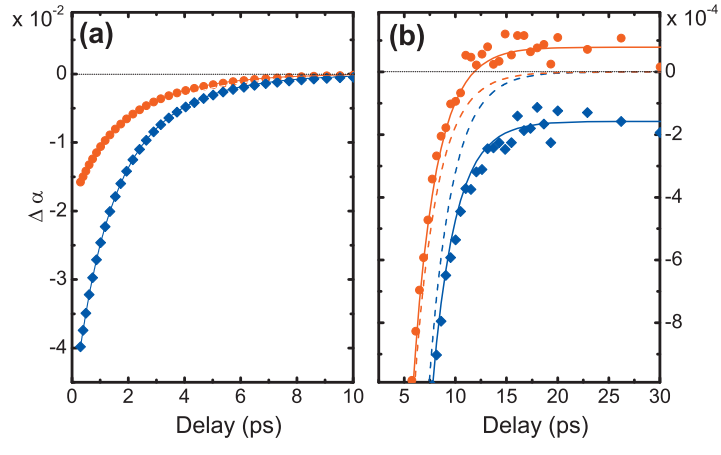

FIG. 5. The parallel (blue diamonds) and perpendicular (red circles) signals measured for a solution of TMAO in HDO: $\mathrm{H}_{2} \mathrm{O}$ of $w=0.2$. (b) is a zoom-in of (a). The full lines are fits using the model described in the text. The dashed lines in (b) represent the calculated parallel and the perpendicular signals after correction for the increased mobility of the relaxing O-D oscillators (see text).

\section{ANALYSIS}

\section{A. 2D-IR experiments}

The spectral dynamics measured in the 2D-IR experiments is quantitatively analyzed by plotting the frequency $\omega_{3, \max }$ of the maximum probe response as a function of the excitation frequency $\omega_{1}$ and by calculating the slope of the $\omega_{3, \max }\left(\omega_{1}\right)$ function for different values of the waiting time $T,^{23}$

$$
S(T)=\left.\frac{\partial \omega_{3, \max }}{\partial \omega_{1}}\right|_{T}
$$

For the solutions of TBA and TMAO, $S(T)$ shows a fast initial decay and then appears to saturate at a nonzero level. This level increases with increasing concentration of the solute. For all concentrations, we fit the slope $S(T)$ with the following expression:

$$
S(T)=S(0)\left(A_{1} e^{-T / \tau_{1}}+A_{2} e^{-T / \tau_{2}}+A_{0}\right)
$$

where $A_{1}$ and $A_{2}$ represent the spectral dynamics that are also observed for pure HDO: $\mathrm{D}_{2} \mathrm{O} . A_{0}$ represents the fraction of $\mathrm{O}-\mathrm{H}$ groups showing slow spectral diffusion. We perform a global fit of Eq. (3) to all measured solutions, with the restriction that the parameters $\tau_{1}$ and $\tau_{2}$ are the same for all solutions. We find time constants $\tau_{1}=0.15 \mathrm{ps}$ and $\tau_{2}$ $=0.78 \mathrm{ps}$, which agree very well with the literature data for pure HDO: $\mathrm{D}_{2} \mathrm{O} .{ }^{34}$ The $\tau_{1}$ process has been assigned to librational motions of the $\mathrm{O}-\mathrm{H}$ groups that keep the $\mathrm{O}-\mathrm{H} \cdots \mathrm{O}$ hydrogen bond intact. The $\tau_{2}$ process has been assigned to collective reorganizations of the hydrogen-bond network. In Fig. 6, the amplitudes $A_{0}, A_{1}$, and $A_{2}$ are plotted as a function of the concentration $w$ for TBA and TMAO. For both solutions, the amplitude $A_{1}$ of the fast component shows little dependence on the concentration. The amplitude $A_{2}$, representing the diffusive part of the bulk water spectral diffusion, strongly decreases with concentration, while the amplitude $A_{0}$ of the slow spectral diffusion component increases. The increase of $A_{0}$ is complementary to the decrease of $A_{2}$. 


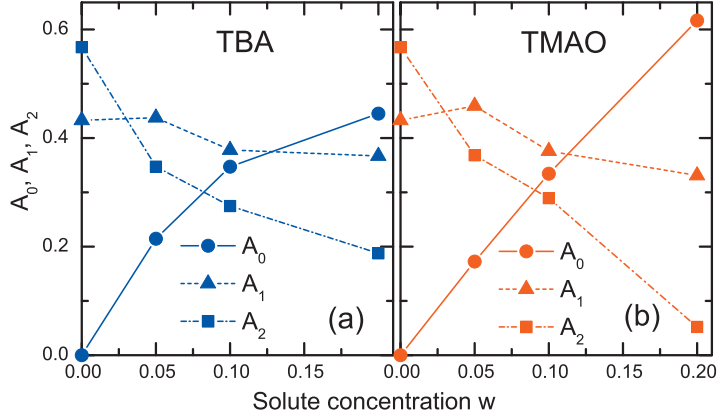

FIG. 6. The amplitudes $A_{0}$ (circles), $A_{1}$ (triangles), and $A_{2}$ (squares) as a function of the concentration for solutions of TBA (upper panel) and TMAO (lower panel). The values of $A_{0}, A_{1}$, and $A_{2}$ are obtained from fits to the 2D-IR data using Eq. (3).

\section{B. Polarization-resolved pump-probe experiments}

The overshoot in $\Delta \alpha_{\perp}(t)$ shown in Fig. 5 implies that more O-D oscillators are oriented perpendicular to the pump polarization than parallel to the pump polarization. This difference can be explained from an increased orientational mobility of the O-D groups that undergo vibrational excitation and relaxation. In the vibrational relaxation process, a large amount of energy is dissipated into nearby low-frequency modes (hydrogen bonds, librational modes), making the O-D group temporarily less strongly hydrogen-bonded and more mobile. Due to the faster reorientation, the anisotropic distribution of the O-D groups that were excited equilibrates faster than the anisotropic distribution of the depleted molecules in the ground state. Hence, at the time when the distribution of the excited and relaxed molecules is already nearly isotropic, the depletion of the ground state still shows a strong anisotropy. Therefore, at that time, there will be more O-D groups perpendicular to the pump polarization than parallel to the pump polarization.

The accelerated reorientation of the excited HDO molecules has been observed before in polarization-resolved pump-probe measurements of the $\mathrm{O}-\mathrm{H}$ stretch vibration of $\mathrm{HDO}$ in $\mathrm{D}_{2} \mathrm{O} .{ }^{35}$ In this earlier study, the observed transient anisotropy could be well described by assigning a faster reorientation to the intermediate state in the relaxation pathway. Here, we employ the same procedure as in Ref. 35 to model the dynamics of $\Delta \alpha_{\|}(T)$ and $\Delta \alpha_{\perp}(T)$. In this procedure, we need to include the dynamics of the anisotropy of the excited O-D oscillators (before the relaxation to the intermediate state) and that of the depleted molecules in the ground state. The anisotropy of aqueous solutions of amphiphilic solutes has been found to show a biexponential decay, with a fast component having a time constant of $2.5 \mathrm{ps}$, similar to what is observed for the reorientation of pure $\mathrm{HDO}: \mathrm{D}_{2} \mathrm{O},{ }^{29}$ and a slow component with a time constant $>10$ ps. $^{12-14}$ The amplitude of the slow component was observed to increase with the solute concentration. This latter component was assigned to the reorientation of water in the hydration shell of the hydrophobic part of the solute. $^{12-14}$

We describe the anisotropy in a similar manner as in Refs. $12-14$, i.e., with a fast component with a reorientation time constant similar to that of bulk liquid water and a slow component representing the reorientation of water molecules in the hydration shells of the solutes. We take the reorientation time constant of the water molecules in the hydration shell to be infinitely large to allow for a quantitative comparison with the hydrogen-bond dynamics measured in the 2D-IR experiments. The orientational dynamics of the hydration shell water molecules is thus described as an offset in the decay of the anisotropy parameter $R(T)$,

$$
R(T)=R(0)\left(B_{1} e^{-T / \tau_{\text {or }}}+B_{0}\right),
$$

where $B_{1}$ represents the contribution to the observed anisotropy of the water molecules showing fast orientational dynamics with time constant $\tau_{\text {or }}$ and $B_{0}$ represents the contribution of water molecules showing a much slower reorientation time constant. For all the studied TBA solutions, the time constant $\tau_{\text {or }}$ has a value of $2.5 \mathrm{ps}$, similar to the reorientation time constant of 2.5 ps of the $\mathrm{O}-\mathrm{D}$ vibration of pure $\mathrm{HDO}: \mathrm{D}_{2} \mathrm{O}$. For TMAO, the time constant of the fast reorienting water molecules has been observed to become somewhat shorter with increasing concentration. ${ }^{36}$ Here, we take $\tau_{\text {or }}$ equal to $2.5 \mathrm{ps}$ at all concentrations to limit the number of parameters in the fits. We find that with increasing concentration, the fraction $B_{1}$ decreases while the fraction $\mathrm{B}_{0}$ increases.

An example of the fit of the model to $\Delta \alpha_{\|}(t)$ and $\Delta \alpha_{\perp}(t)$ is shown in Fig. 5. From the model, we directly obtain the time-dependent anisotropy of the excitation of the O-D groups. In Fig. 7, we show the delay dependence of the anisotropy for different concentrations of TBA and TMAO. In
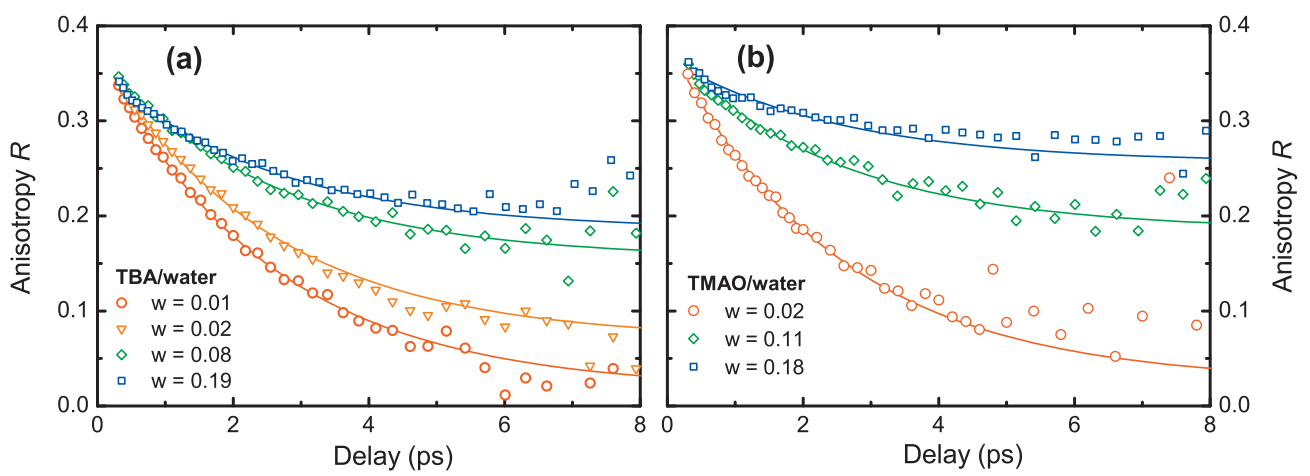

FIG. 7. Anisotropy decays of the excitation of the O-D stretch vibration of the HDO molecules for four different solutions of TBA (left panel) and three different solutions of TMAO (right panel) in $\mathrm{HDO}: \mathrm{H}_{2} \mathrm{O}$. The lines result from a fit to Eq. (4). 


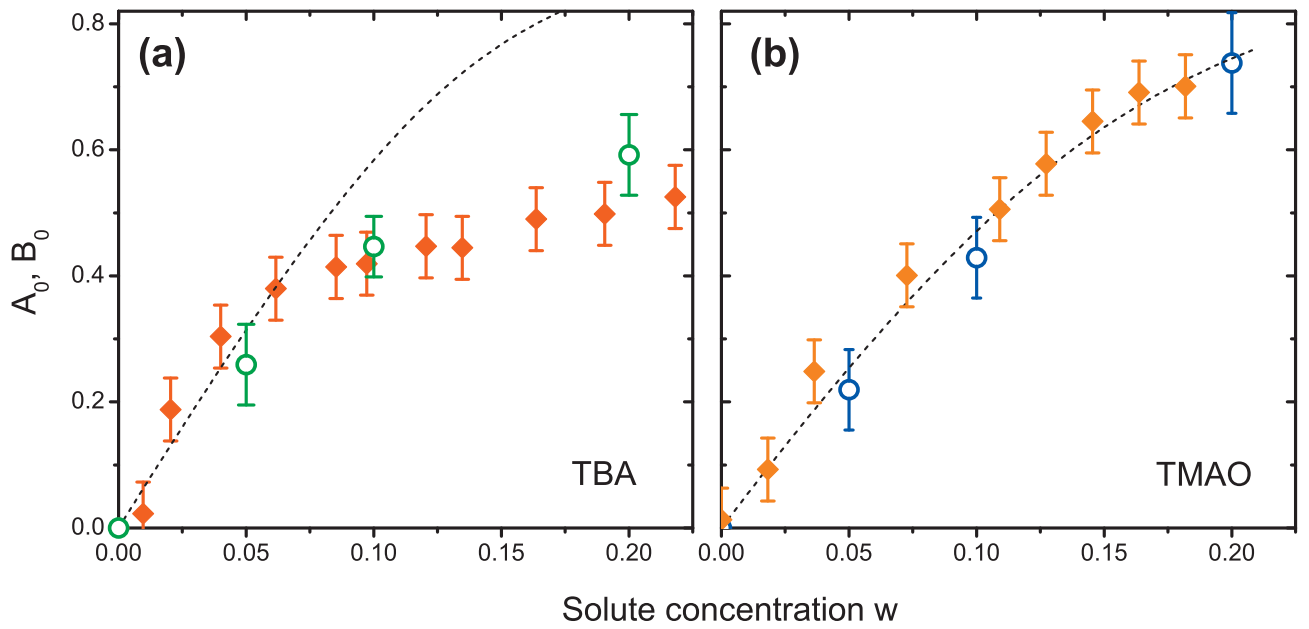

FIG. 8. The amplitude $A_{0}$ (open symbols) of the HDO molecules showing slow spectral diffusion and the fraction $B_{0}$ of slowly reorienting HDO (filled symbols) as a function of the solute concentration $w$ for solutions of TBA (left panel) and TMAO (right panel). To facilitate the comparison, the values of $A_{0}$ are multiplied by a scaling factor of 1.23 that is the same at all concentrations. The values of $A_{0}$ are obtained from fits to the 2D-IR data using Eq. (3), and the values of $B_{0}$ are obtained from fits to the anisotropy decay curves using Eq. (4). The dashed lines result from fits of $A_{0}$ and $B_{0}$ to Eq. (8) for the TBA data at low concentrations $(w<0.05)$ and for the TMAO data at all concentrations.

Fig. 8, the fraction of slowly reorienting water $B_{0}$ is shown as a function of the concentration $w$ for both TBA and TMAO. At low concentrations, $B_{0}$ increases linearly with concentration with a slope that is similar for the solutions of TBA and TMAO. In the same figure, we also present the amplitudes $A_{0}$ obtained from the 2D-IR experiments.

\section{DISCUSSION}

Figure 8 shows that there is a perfect correlation between the concentration dependencies of the fraction $A_{0}$ of HDO molecules showing slow spectral diffusion and the fraction $B_{0}$ of HDO showing slow reorientation. For both TBA and TMAO, $A_{0}$ and $B_{0}$ show the same linear increase at low concentrations $(w \leq 0.05)$ and the same saturation behavior at high concentrations $(w>0.05)$. This strong correlation indicates that the slowing down of the spectral diffusion and the molecular reorientation have a common origin. As the reorientation and spectral diffusion both rely on the transient formation of bifurcated hydrogen-bonded structures, the slowing down can be explained from the hindrance of the formation of bifurcated hydrogen bonds in the hydration shells of the solutes. ${ }^{33}$

At low solute concentration $(w \leq 0.05), A_{0}$ and $B_{0}$ increase with similar slopes for TBA and TMAO (Fig. 8), which shows that these solutes have a similar effect on the hydrogen bond and orientational dynamics of water at low concentrations. This finding differs from the results of recent classical molecular dynamics simulations in which it was found that TMAO has a much stronger effect on the water dynamics than TBA. ${ }^{37}$ This difference was explained from the fact that water has a much stronger interaction with the hydrophilic $\mathrm{N}^{+} \mathrm{O}^{-}$group of TMAO than with the hydrophilic hydroxyl group of TBA. Here, we observe that the effects of TMAO and TBA on the water dynamics are in fact quite similar, which suggests that the difference in the interaction with the hydrophilic groups is overwhelmed by another effect, i.e., the hydration of the hydrophobic parts of the mol- ecules. This notion agrees with the results of previous NMR studies, ${ }^{7,8,10,11}$ femtosecond anisotropy measurements, ${ }^{12-14}$ and dielectric relaxation studies. ${ }^{38}$ In all these studies, it was found that the effect of the solute on the dynamics of water scales with the size of the hydrophobic part of the solute molecule.

Figure 8 shows that for both TBA and TMAO, the number of slow hydroxyl groups per solute molecule decreases with increasing solute concentration. This saturation can be due to several effects including the competition for solvating water molecules (more solvation sites available than there are water molecules) and aggregation. If there is no specific interaction between the solute molecules (no aggregation), the concentrations of fast and slow hydroxyl groups can be described using a chemical equilibrium,

$$
\frac{[\mathrm{OH} \cdots \mathrm{S}]}{[\mathrm{OH}][\mathrm{S}]}=K_{h},
$$

where $[\mathrm{OH} \cdots \mathrm{S}]$ is the concentration of slow hydroxyl groups in the hydration shell of a solute molecule, $[\mathrm{OH}]$ is the concentration of hydroxyl groups showing bulklike fast reorientation, and $[\mathrm{S}]$ is the concentration of open positions for a hydroxyl group in a solute hydration shell. The total concentration of (occupied and unoccupied) solvation shell sites $([\mathrm{OH} \cdots \mathrm{S}]+[\mathrm{S}])$ is equal to $C_{\mathrm{s}} N_{\mathrm{s}}$, with $N_{\mathrm{s}}$ being the number of possible sites for a slow hydroxyl group in the hydration shell of a solute molecule. Assuming that the density of all solutions equals $1 \mathrm{~g} / \mathrm{cm}^{3}$, the concentration $C_{\mathrm{s}}$ is given by

$$
C_{\mathrm{s}}=\frac{1000 w}{M_{\mathrm{s}} w+M_{\mathrm{H}_{2} \mathrm{O}}},
$$

where $M_{\mathrm{s}}$ is the molecular weight of the solute molecule and $M_{\mathrm{H}_{2} \mathrm{O}}$ is the molecular weight of water $(=18)$. The total concentration of the hydroxyl groups $C_{\mathrm{OH}}(=[\mathrm{OH} \cdots \mathrm{S}]+[\mathrm{OH}])$ also depends on $w$ and is given by 


$$
C_{\mathrm{OH}}=2 \frac{1000-C_{\mathrm{s}} M_{\mathrm{s}}}{M_{\mathrm{H}_{2} \mathrm{O}}} .
$$

Using that the concentration of unoccupied hydration shell sites $[\mathrm{S}]=C_{\mathrm{S}} N_{\mathrm{s}}-[\mathrm{OH} \cdots \mathrm{S}]$ and fast, bulklike hydroxyl groups $[\mathrm{OH}]=C_{\mathrm{OH}^{-}}-[\mathrm{OH} \cdots \mathrm{S}]$, we can solve Eq. (5) for the concentration of slow hydroxyl groups $[\mathrm{OH} \cdots \mathrm{S}]$,

$$
\begin{aligned}
{[\mathrm{OH} \cdots \mathrm{S}]=} & \frac{1}{2}\left\{C_{\mathrm{OH}}+C_{\mathrm{s}} N_{\mathrm{s}}+K_{h}^{-1}\right. \\
& \left.-\sqrt{\left(C_{\mathrm{OH}}+C_{\mathrm{s}} N_{\mathrm{s}}+K_{h}^{-1}\right)^{2}-4 C_{\mathrm{OH}} C_{\mathrm{s}} N_{\mathrm{s}}}\right\} .
\end{aligned}
$$

The fraction of slow water $f_{\mathrm{s}}$ is given by $[\mathrm{OH} \cdots \mathrm{S}] / C_{\mathrm{OH}}$.

For TMAO, the concentration dependence of the fraction of slow hydroxyl groups can be well described with the above equation using $N_{\mathrm{s}}=14$ and $K_{h}=0.06 \mathrm{M}^{-1}$. For a solution with $w=0.02$, this gives $f_{\mathrm{s}}=0.12$ and 12 slow $\mathrm{O}-\mathrm{H}$ groups per TMAO molecule, while for a solution with $w$ $=0.2$, the slow fraction $f_{\mathrm{s}}=0.72$ and there are 7 slow $\mathrm{O}-\mathrm{H}$ groups per TMAO molecule. The fact that for TMAO the concentration dependence of the slow fraction can be well described with Eq. (8) shows that for this solute the saturation is due to the competition of the solvation sites for solvating water molecules and not due to the aggregation of the solute molecules. The absence of clustering for TMAO agrees with the results of molecular dynamics simulations. ${ }^{39}$

For TBA, the saturation effect is much stronger, indicating that these molecules cluster. For TBA, only the low concentration region can be fitted well to Eq. (8), using the values $N_{\mathrm{s}}=16$ and $K_{h}=0.1 M^{-1}$. Here, $K_{h}$ had to be fixed because the initial slope depends on the product $N_{\mathrm{s}} K_{h}$ only. These two parameters can only be disentangled if the fit would also describe the saturation behavior at high values of $w$, which was not possible for TBA. For a solution with $w$ $=0.02$, we find (Fig. 8) $f_{\mathrm{s}}=0.14$ and 14 slow $\mathrm{O}-\mathrm{H}$ groups per TBA molecule. For $w>0.05$, the observed fraction of slow HDO molecules increasingly deviates from the curve calculated with Eq. (8), and the fraction of slow HDO molecules saturates at a maximum of $\sim 0.55$. In the case of a chemical equilibrium, the fraction of slow HDO would ultimately increase to 1 . The presence of a maximum of the fraction of slow HDO for TBA implies that beyond a certain concentration $(w \sim 0.1)$ all additional TBA are embedded by other TBA molecules, i.e., incorporated in the TBA aggregates.

Aggregation of TBA has been observed before in molecular dynamics simulations (MD) and neutron scattering studies. ${ }^{16,4,20,39,40}$ MD simulations showed that for a mole fraction of 0.08 , clusters of 3-4 TBA molecules were formed. ${ }^{40}$ Neutron scattering studies for a TBA solution of $0.06 \mathrm{~mol}$ fraction also showed that clusters are formed with a size of $3 \pm 0.5$ TBA molecules. ${ }^{16}$

The maximum fraction of slowly reorienting HDO molecules is $\sim 0.55$, which implies that even for very highly concentrated TBA-water mixtures, $\sim 45 \%$ of the water retains its bulklike properties. These water molecules are likely located in nanoclusters/droplets of water, embedded between the TBA aggregates. Neutron scattering studies indeed indicated that small water nanoclusters are formed in highly con- centrated TBA solutions. For a solution with a mole fraction of 0.86 , TBA clusters of 2-3 water molecules were observed. ${ }^{41}$

The absence of aggregation for TMAO solutions is quite surprising in view of the fact that TMAO is an osmolyte that stimulates the folding of proteins. This means that TMAO stimulates the hydrophobic clustering of other dissolved amphiphilic molecules, but apparently does not stimulate its own hydrophobic aggregation. The difference in the aggregation behavior of TBA and TMAO is probably not associated with the hydrophobic parts of the molecules, as these parts are very similar. The lack of aggregation for TMAO is thus likely the result of differences in the interactions between the water solvent and the hydrophilic part of the TBA and TMAO molecules. ${ }^{39}$ The oxygen atom of TMAO has three lone pairs and can accept more hydrogen bonds from the surrounding water molecules than the two lone pairs of the oxygen atom of the alcohol hydroxyl group of TBA. The oxygen atom of TMAO is also more exposed than the oxygen atom of TBA. Hence, there will be more and stronger hydrogen bonds to the hydrophilic part of TMAO than to the hydroxyl group of TBA, as is evidenced by the infrared absorption spectrum (Fig. 1). The hydrogen-bond structure of water molecules near the hydrophilic group of TMAO thus prevents the clustering of the hydrophobic parts of the TMAO molecules, while stimulating the hydrophobic aggregation of other dissolved molecules.

\section{CONCLUSIONS}

We studied the aggregation behavior of solute molecules in aqueous solutions of TBA and TMAO using femtosecond two-dimensional midinfrared spectroscopy and polarizationresolved femtosecond midinfrared spectroscopy. We measured the spectral dynamics of the $\mathrm{O}-\mathrm{H}$ stretch vibration of HDO in solutions with $\mathrm{D}_{2} \mathrm{O}$ as the solvent and the orientational dynamics of the $\mathrm{O}-\mathrm{D}$ vibration of HDO in solutions with $\mathrm{H}_{2} \mathrm{O}$ as the solvent. For both solutions, a fraction of the HDO molecules is observed to show a much slower hydrogen bond and reorientation dynamics than the HDO molecules in pure HDO: $\mathrm{D}_{2} \mathrm{O} / \mathrm{H}_{2} \mathrm{O}$. At low concentrations $(w$ $\leq 0.05$ ), the fraction of slow HDO molecules rises linearly with the concentration. These increases have similar slopes for TBA and TMAO, showing that the effect of these solutes on the water dynamics is governed by their similar hydrophobic parts and not by their different hydrophilic parts.

At high concentrations $(w>0.05)$, the increase of the fraction of slow HDO molecules with concentration saturates. For TMAO, the saturation behavior can be well described with a chemical equilibrium between solvation shell sites and bulk sites for water hydroxyl groups, which implies that the saturation is not due to aggregation but results from the competition between the solvation shell and the bulk. For TBA, the saturation behavior is much stronger, and there is a maximum fraction of slow water, which indicates that TBA forms aggregates. This finding agrees with the previous results from molecular dynamics simulations and neutron scattering studies. The maximum fraction of slow HDO of $\sim 0.55$ of TBA solutions implies that $\sim 45 \%$ of the water 
retains its bulklike character in highly concentrated TBA solutions. This bulklike water is likely contained in water nanodroplets that are embedded by the TBA aggregates.

\section{ACKNOWLEDGMENTS}

This work was part of the research program of the "Stichting voor Fundamenteel Onderzoek der Materie (FOM)," which was financially supported by the "Nederlandse organisatie voor Wetenschappelijk Onderzoek (NWO)." M.S.P. thanks A. Tarasov ("Consensus," Moscow) for his hospitality during the volcano-caused European air collapse in April 2010, while he was working on the manuscript. We also acknowledge the financial support from the Villum Kann Rasmussen Foundation.

${ }^{1}$ H. S. Frank and M. W. Evans, J. Chem. Phys. 13, 507 (1945).

${ }^{2}$ A. K. Soper and J. L. Finney, Phys. Rev. Lett. 71, 4346 (1993).

${ }^{3}$ J. Turner and A. K. Soper, J. Chem. Phys. 101, 6116 (1994).

${ }^{4}$ D. T. Bowron, J. L. Finney, and A. K. Soper, J. Phys. Chem. B 102, 3551 (1998).

${ }^{5}$ S. Dixit, J. Crain, W. C. K. Poon, J. L. Finney, and A. K. Soper, Nature (London) 416, 829 (2002).

${ }^{6}$ M. Nakahara, C. Wakai, Y. Yoshimoto, and N. Matubayashi, J. Phys. Chem. 100, 1345 (1996).

${ }^{7}$ Y. Ishihara, S. Okouchi, and H. Uedaira, J. Chem. Soc., Faraday Trans. 93, 3337 (1997).

${ }^{8}$ K. Fumino, K. Yukiyasu, A. Shimizu, and Y. Taniguchi, J. Mol. Liq. 75 1 (1998).

${ }^{9}$ K. Yoshida, K. Ibuki, and M. Ueno, J. Chem. Phys. 108, 1360 (1998).

${ }^{10}$ A. Shimizu, K. Fumino, K. Yukiyasu, and Y. Taniguchi, J. Mol. Liq. 85, 269 (2000).

${ }^{11}$ J. Qvist and B. Halle, J. Am. Chem. Soc. 130, 10345 (2008).

${ }^{12}$ Y. L. A. Rezus and H. J. Bakker, Phys. Rev. Lett. 99, 148301 (2007)

${ }^{13}$ Y. L. A. Rezus and H. J. Bakker, J. Phys. Chem. A 112, 2355 (2008).

${ }^{14}$ C. Petersen, K.-J. Tielrooij, and H. J. Bakker, J. Chem. Phys. 130, 214511 (2009)

${ }^{15}$ D. Laage, G. Stirneman, and J. T. Hynes, J. Phys. Chem. B 113, 2428 (2009).

${ }^{16}$ D. T. Bowron, A. K. Soper, and J. L. Finey, J. Chem. Phys. 114, 6203
(2001).

${ }^{17}$ S. Dixit, A. K. Soper, J. L. Finney, and J. Crain, Europhys. Lett. 59, 377 (2002)

${ }^{18}$ A. B. Roney, B. Space, E. W. Castner, R. L. Napoleon, and P. B. Moore, J. Phys. Chem. B 108, 7389 (2004).

${ }^{19}$ L. Dougan, S. P. Bates, R. Hargreaves, J. P. Fox, J. Crain, J. L. Finney, V. Reat, and A. K. Soper, J. Chem. Phys. 121, 6456 (2004).

${ }^{20}$ D. T. Bowron and J. L. Finney, J. Phys. Chem. B 111, 9838 (2007).

${ }^{21}$ J. B. Asbury, T. Steinel, C. Stromberg, S. A. Corcelli, C. P. Lawrence, J. L. Skinner, and M. D. Fayer, J. Phys. Chem. A 108, 1107 (2004).

${ }^{22}$ J. J. Loparo, S. T. Roberts, and A. Tokmakoff, J. Chem. Phys. 125, 194521 (2006).

${ }^{23}$ K. Kwak, S. Park, I. J. Finkelstein, and M. D. Fayer, J. Chem. Phys. 127, 124503 (2007).

${ }^{24}$ K. Lazonder, M. S. Pshenichnikov, and D. A. Wiersma, Opt. Lett. 31, 3354 (2006).

${ }^{25}$ S. Woutersen, U. Emmerichs, and H. J. Bakker, Science 278, 658 (1997).

${ }^{26}$ G. Gallot, S. Bratos, S. Pommeret, N. Lascoux, J.-C. Leicknam, M. Kozinski, W. Amir, and G. M. Gale, J. Chem. Phys. 117, 11301 (2002).

${ }^{27}$ T. Steinel, J. B. Asbury, J. Zheng, and M. D. Fayer, J. Phys. Chem. A 108, 10957 (2004).

${ }^{28}$ C. J. Fecko, J. J. Loparo, S. T. Roberts, and A. Tokmakoff, J. Chem. Phys. 122, 054506 (2005).

${ }^{29}$ Y. L. A. Rezus and H. J. Bakker, J. Chem. Phys. 123, 114502 (2005).

${ }^{30}$ S. Woutersen and H. J. Bakker, Nature (London) 402, 507 (1999).

${ }^{31}$ S. Yeremenko, A. Baltuska, F. de Haan, M. S. Pshenichnikov, and D.A. Wiersma, Opt. Lett. 27, 1171 (2002).

${ }^{32}$ M. J. Tauber, R. A. Mathies, X. Y. Chen, and S. E. Bradforth, Rev. Sci. Instrum. 74, 4958 (2003)

${ }^{33}$ A. A. Bakulin, C. Liang, T. La Cour Jansen, D. A. Wiersma, H. J. Bakker, and M. S. Pshenichnikov, Acc. Chem. Res. 42, 1229 (2009).

${ }^{34}$ C. J. Fecko, J. D. Eaves, J. J. Loparo, A. Tokmakoff, and P. L. Geissler, Science 301, 1698 (2003).

${ }^{35}$ Y. L. A. Rezus and H. J. Bakker, J. Chem. Phys. 125, 144512 (2006).

${ }^{36}$ Y. L. A. Rezus and H. J. Bakker, J. Phys. Chem. B 113, 4038 (2009).

${ }^{37}$ G. Stirnemann, J. T. Hynes, and D. Laage, J. Phys. Chem. B 114, 3052 (2010).

${ }^{38}$ S. Schrödle, R. Buchner, and W. Kunz, J. Phys. Chem. B 108, 6281 (2004).

${ }^{39}$ S. Paul and G. N. Patey, J. Phys. Chem. B 110, 10514 (2006).

${ }^{40}$ P. G. Kusalik, A. P. Lyubartsev, D. L. Bergman, and A. Laaksonen, J. Phys. Chem. B 104, 9533 (2000).

${ }^{41}$ D. T. Bowron and S. Diaz Moreno, J. Chem. Phys. 117, 3753 (2002). 